Cahiers de géographie du Québec

\title{
Les interactions entre cultures sportives de montagne et territoires : un état des lieux de la recherche française depuis 1990
}

\section{Sporting Cultures and Territories: An Inventory of French} Research Since the 1990s

\author{
Philippe Bourdeau, Jean Corneloup, Pascal Mao et Éric Boutroy
}

Volume 48, numéro 133, avril 2004

URI : https://id.erudit.org/iderudit/009761ar

DOI : https://doi.org/10.7202/009761ar

Aller au sommaire du numéro

Éditeur(s)

Département de géographie

ISSN

0007-9766 (imprimé)

1708-8968 (numérique)

Découvrir la revue

Citer cet article

Bourdeau, P., Corneloup, J., Mao, P. \& Boutroy, É. (2004). Les interactions entre cultures sportives de montagne et territoires : un état des lieux de la recherche française depuis 1990. Cahiers de géographie du Québec, 48(133), 33-46.

https://doi.org/10.7202/009761ar
Résumé de l'article

Les relations entre cultures sportives et territoires constituent un champ de recherche en plein essor en France depuis le début des années 1990. Une grille de lecture socio-spatiale est appliquée à un corpus de textes géographiques, sociologiques, historiques et ethnologiques portant sur cette question. Cette démarche est orientée selon quatre registres conceptuels (espace, spatialité, territoire, territorialité) et interactionnels (physique, fonctionnel, organisationnel, existentiel), qui mettent en perspective l'orientation thématique et le point de vue théorique des travaux pris en compte. Cet état des lieux des représentations scientifiques permet d'expliciter le phénomène de co-construction temporelle des cultures sportives et de leurs espaces d'action. 


\title{
Les interactions entre cultures sportives de montagne et territoires : un état des lieux de la recherche française depuis 1990
}

\author{
Philippe Bourdeau, Jean Corneloup, Pascal Mao et Éric Boutroy \\ Institut de Géographie Alpine \\ Université Joseph Fourier \\ philippe.bourdeau@ujf-grenoble.fr \\ j.corneloup@libertysurf.fr \\ pascal.mao@sportsnature.org \\ e.boutroy@libertysurf.fr
}

\section{Résumé}

Les relations entre cultures sportives et territoires constituent un champ de recherche en plein essor en France depuis le début des années 1990. Une grille de lecture sociospatiale est appliquée à un corpus de textes géographiques, sociologiques, historiques et ethnologiques portant sur cette question. Cette démarche est orientée selon quatre registres conceptuels (espace, spatialité, territoire, territorialité) et interactionnels (physique, fonctionnel, organisationnel, existentiel), qui mettent en perspective l'orientation thématique et le point de vue théorique des travaux pris en compte. Cet état des lieux des représentations scientifiques permet $\mathrm{d}^{\prime}$ expliciter le phénomène de co-construction temporelle des cultures sportives et de leurs espaces d'action.

Mots-clés : culture sportive, espace, spatialité, territoires, territorialité, France, montagne, sports de nature, alpinisme, Alpes, escalade.

\begin{abstract}
Sporting Cultures and Territories: An Inventory of French Research Since the 1990s

Since the early 1990s, the study of relationships between sporting cultures and territories has become a rapidly growing field of research. A socio-spatial analysis is applied to a corpus of geographical, sociological, historical and ethnological texts relating to this question. Research is based on two types of frameworks: conceptual (space, spatiality, territory, territoriality) and inter-actional (physical, functional, organisational, existential), giving a thematic and theoretical perspective to this work. This inventory of different forms of scientific representation seeks to clarify the concept of the temporalized coconstruction of sporting cultures and their action spaces.
\end{abstract}

Key Words: $\quad$ sporting cultures, space, spatiality, territories, territoriality, France, mountain, Alps, outdoor sports, mountain climbing, mountaineering. 


\section{ENTRÉE EN MATIÈRE : ÉLÉMENTS DE QUESTIONNEMENT}

L'approche proposée vise à brosser un état des lieux de la recherche française sur les interactions entre cultures sportives ${ }^{1}$ et espaces de pratique des sports de nature et de montagne. À partir de travaux sociologiques, ethnologiques, géographiques et historiques effectués depuis le début des années 1990, il s'agit d'interroger les représentations scientifiques de l'influence de l'espace sur les pratiques et les cultures sportives, en même temps que les représentations de la production des formes géo-sportives (sites, itinéraires, parcours, "spots »...) découlant des pratiques et des cultures sportives. Dans le même temps, nous esquissons un certain nombre de pistes de recherche qui s'annoncent comme fécondes et les situons par rapport à cet « état des lieux » de la connaissance. Cette démarche s'inscrit dans une perspective de mobilisation de référents socio-spatiaux, indicateurs pertinents et opérateurs de la création et de l'actualisation de pratiques, de représentations, d'imaginaires, dont la non-indifférentiation n'est pas seulement produite, mais aussi agissante (Chivallon, 1998), productrice et médiatrice (Augustin, 2000).

La synthèse proposée cherche à saisir non seulement les thématiques scientifiques explicitant les interactions entre les cultures sportives et les espaces de nature, mais aussi les prises de position théoriques, méthodologiques et heuristiques qui les sous-tendent. Notre démarche est orientée selon quatre registres conceptuels (espace, spatialité, territoire, territorialité) et interactionnels (physique, fonctionnel, organisationnel, existentiel) qui structurent la grille de lecture sociospatiale à partir de laquelle les travaux et les pistes de recherche pris en compte sont présentés (tableau 1). Ces registres se sont pas retenus ici comme des objets géographiques ni comme les étapes d'un processus de construction socio-spatiale, mais plutôt comme des échelles de saisie à la fois distinctes et complémentaires permettant de caractériser, à partir de différentes variables scalaires, méthodologiques et théoriques, les différentes logiques d'intelligibilité mises en oeuvre de manière explicite ou implicite dans les travaux pris en compte.

Tableau 1 Une grille de lecture socio-spatiale des interactions entre cultures sportives de nature et espaces de pratiques

\begin{tabular}{|c|c|c|c|c|c|c|}
\hline $\begin{array}{l}\text { Échelle socio- } \\
\text { géographique }\end{array}$ & $\begin{array}{c}\text { Référent } \\
\text { socio-spatial }\end{array}$ & $\begin{array}{c}\text { Référent } \\
\text { sociologique } \\
\text { privilégié }\end{array}$ & $\begin{array}{c}\text { Objet } \\
\text { géographique } \\
\text { privilégié }\end{array}$ & $\begin{array}{c}\text { Processus-type } \\
\text { étudié }\end{array}$ & $\begin{array}{c}\text { Dominante } \\
\text { de l'approche }\end{array}$ & $\begin{array}{c}\text { Registre } \\
\mathrm{d}^{\prime} \text { interaction } \\
\text { culture-espaces }^{2}\end{array}$ \\
\hline macro & $\begin{array}{c}\text { Espace } \\
\text { «espace } \\
\text { support» }\end{array}$ & $\begin{array}{l}\text { variable } \\
\text { sociale } x\end{array}$ & $\begin{array}{l}\text { État } \\
\text { région }\end{array}$ & $\begin{array}{l}\text { localisation } \\
\text { hiérarchie }\end{array}$ & $\begin{array}{l}\text { descriptive } \\
\text { cartographique } \\
\text { quantitative } \\
\text { structurelle }\end{array}$ & physique \\
\hline \multirow[t]{2}{*}{ meso } & $\begin{array}{l}\text { Spatialité } \\
\text { "espace } \\
\text { d'action" }\end{array}$ & individus & $\begin{array}{c}\text { massif } \\
\text { vallée } \\
\text { bassin sportif }\end{array}$ & $\begin{array}{c}\text { fréquentation } \\
\text { cheminements } \\
\text { flux } \\
\text { pratiques }\end{array}$ & $\begin{array}{l}\text { structurelle } \\
\text { actantielle }\end{array}$ & fonctionnel \\
\hline & $\begin{array}{l}\text { Territoire } \\
\text { « espace } \\
\text { produit }\end{array}$ & $\begin{array}{l}\text { groupes } \\
\text { acteurs }\end{array}$ & $\begin{array}{l}\text { vallée } \\
\text { station }\end{array}$ & $\begin{array}{l}\text { développement, } \\
\text { stratégie, conflit } \\
\text { acteurs }\end{array}$ & $\begin{array}{c}\text { systémique } \\
\text { constructiviste }\end{array}$ & organisationnel \\
\hline micro & $\begin{array}{l}\text { Territorialité } \\
\text { "espace vécu" }\end{array}$ & $\begin{array}{l}\text { pratiquant } \\
\text { personne }\end{array}$ & $\begin{array}{l}\text { Site } \\
\text { « spot» }\end{array}$ & $\begin{array}{c}\text { vécu, expérience } \\
\text { proxémie } \\
\text { culture, identité }\end{array}$ & $\begin{array}{c}\text { interactionnelle } \\
\text { qualitative } \\
\text { phénoménologique }\end{array}$ & existentiel \\
\hline
\end{tabular}




\title{
L'ESPACE DES PRATIQUES SPORTIVES DE NATURE COMME CHAMP D'INTERACTION PHYSIQUE
}

\author{
LE DÉTOUR STRUCTUREL : À LA RECHERCHE DE RÉGULARITÉS \\ ET DE PRINCIPES STRUCTURANTS
}

Le registre spatial constitue le niveau d'entrée qui a été le plus largement mobilisé par les chercheurs français pour décrypter les éléments explicatifs de la structuration des activités sportives de montagne et de nature. La description des sites, des activités, des acteurs et des publics permet d'identifier le champ global des interactions entre cultures sportives et espaces au sein duquel se distribuent les pratiques, à l'image des « variations géographiques du sport » mises en perspective par A. Haumont (1995). Cette approche est plus quantitative que qualitative, plus extérieure qu'intérieure et plus spéculative qu'empirique, ce qui lui confère un caractère que l'on peut qualifier de structurel. On effectue des diagnostics d'espaces sportifs - notamment sur la base d'approches cartographiques - afin d'identifier les régularités et les facteurs discriminants dans les logiques d'apparition et dans les modes de distribution spatiale des pratiques et des équipements ou aménagements sportifs. La géographie du sport développée par J. Praicheux et D. Mathieu (1987), A. Griffond-Boitier (1995), puis par P. Mao et N. Dupuy (2000) est très représentative de cette production de connaissance.

L'identification de principes structurants est ici la base de la démarche poursuivie et s'appuie sur une vision globale et distanciée cherchant à mettre en évidence des « lois », des forces en action... La priorité est donnée à une approche macro sociogéographique des sports de nature (Augustin, 1995, et Pociello, 1995), faisant appel à une lecture qui peut prendre pour objet les pratiques dans leur ensemble ou une pratique particulière, dans la manière dont celle(s)-ci façonne(nt) les espaces de pratique. Les recherches de J. Corneloup et de P. Bourdeau (2001) sur le rôle des "nouvelles » pratiques sportives et de leurs espaces dans la recomposition du système touristique de la vallée de Vallouise durant la période 1980-2000 s'inscrivent dans ce type de perspective.

\section{La prise en compte de facteurs exogènes...}

Plusieurs recherches s'attachent à montrer combien le développement des espaces de pratique est conditionné par des données structurelles et macrosociologiques externes. À ce titre, les travaux de C. Pociello (1995) montrent que l'émergence de fonctions ludiques, récréatives et de spectacle a eu pour effet de recomposer les logiques de développement du sport en modifiant les cultures sportives et la dynamique des espaces de nature. De même, les écrits d'A. Loret (1993) et de C. Pigeassou (2001) montrent que la forte pénétration du secteur marchand participe à la transformation structurelle des espaces sportifs de nature. Alors que le modèle fordiste (Chazaud, 2000) semble dépassé par l'obligation de s'adapter aux nouvelles règles de structuration des espaces touristiques, l'aménagement de nouveaux sites et parcours sportifs s'explique largement par l'action des prestataires et des collectivités territoriales. Enfin, on ne peut négliger le rôle majeur que joue dans la dynamique des pratiques un facteur exogène comme le système juridique, réglementaire et normatif (Servoin, 1993). 


\section{... et de facteurs endogènes}

Pour étudier la dynamique interne des espaces sportifs de nature, certains chercheurs s'appuient sur des études environnementales destinées à évaluer les perturbations des écosystèmes par la fréquentation de sites et d'itinéraires de pratique (Mounet et al., 2000; Franchini, 2000). D'autres recherches montrent en quoi la fréquentation des espaces dépend de l'entropie des sites de pratique selon des cultures sportives historiquement définies. Elles mettent en évidence une dialectique des sites et des cultures sportives en fonction de leur capacité à produire de la diversité, du changement et de l'innovation, faute de quoi ils se co-saturent et perdent de leur attraction. Les travaux de J. Corneloup (1993) expliquent en partie la transformation des pratiques de l'alpinisme durant les années 1970-80 par la saturation d'un modèle de pratique «classique » et par l'apparition de nouvelles conceptions de l'activité permettant de recréer de la diversité spatiale.

\section{DES APPROCHES HISTORIQUES ET CULTURELLES}

Les écrits de J. Corneloup (1993) sur le processus d'émergence de l'alpinisme mettent de l'avant les facteurs qui ont participé à la modification des référents historiques et géographiques sur la vision de la nature et de la montagne. D'un espace "affreux », chaotique et insignifiant marqué par un syncrétisme religieux complexe et par la prédominance de la culture paysanne, la montagne est devenue en quelques siècles un espace sportif dynamisé par des représentations urbaines de la nature. La médiation culturelle des philosophes, des peintres, des écrivains, des mécaniciens-architectes et autres cartographes (Mizrahi et Bourges, 1979) apparaît ici fondamentale pour saisir la transformation des représentations de la montagne (Debarbieux, 1995) et de ses usages socio-spatiaux, notamment sur les plans sportif et touristique.

\section{Le détour par la culture}

Dans la continuité de ces écrits, P. Bourdeau (1998) explique la transformation contemporaine des relations à la montagne et à la nature par l'émergence d'une culture touristique interactive. Le changement dans la déclinaison des usages des espaces et des pratiques s'explique par l'apparition d'une culture de la consommation qui modifie les attentes de la clientèle des montagnes, à la recherche d'une nature plus ludique et plus moderne en matière d'équipement, d'aménagement et de confort. Antérieurement, les écrits d'A. Loret (1995) ont montré comment la dynamique des pratiques de nature est marquée par la transformation des cultures sportives. L'espace est qualifié culturellement dans les manières d'en faire usage, et l'apparition de la culture récréative explique ce renouvellement des relations aux espaces de pratique. La transformation des référents culturels a pour effet de modifier les rapports à l'espace, les codes de jeu et les significations attendues des échanges sportifs avec la nature. Ce phénomène serait marqué par le passage d'une culture numérique (affrontement, conquête, quantification des performances, marquage institutionnel, héroïsme...) à une culture analogique (défi, immersion dans la nature, priorité aux espaces interstitiels, tribalisme...), et 
stimulerait l'invention de nouvelles modalités de pratique (surf, cascade de glace, parapente, saut d'un point fixe ou base jump, kilomètre lancé, ski de bosses...) qui incite à l'exploration de nouveaux espaces et à la production de sites de pratique. Plusieurs des écrits sur le surf dirigés par J.-P. Augustin (1994), dont ceux de J. Diénot et D. Theiller (1999), s'inscrivent dans une perspective parallèle en mettant en évidence le renouvellement des représentations et des références culturelles en matière de loisirs sportifs de nature depuis le milieu des années 1980.

En prenant l'exemple significatif du champ des pratiques de l'escalade, les écrits de J. Corneloup (1993) ont bien montré que les années 1970-1980 ont été marquées par un mouvement culturel ayant eu pour effet de modifier la communication avec les espaces de la nature. Le passage de l'alpinisme comme pratique dominante durant la modernité (1850-1975) aux activités d'escalade diversifiées caractéristiques de la post-modernité (1975-2000) a fait évoluer l'organisation linéaire des espaces de pratique (des petits sites d'escalade à la haute montagne) vers une organisation circulaire (blocs d'escalade, petites « falaises ${ }^{3}$ », grandes « falaises », cascades gelées, via ferrata ${ }^{4}$, haute montagne, massifs lointains...) qui épouse le modèle du réseau bien plus que celui de la ligne droite, faisant entrer le système des pratiques d'escalade dans une logique du multiple (figure 1).

Figure 1 D'un espace linéaire à un espace réseauté
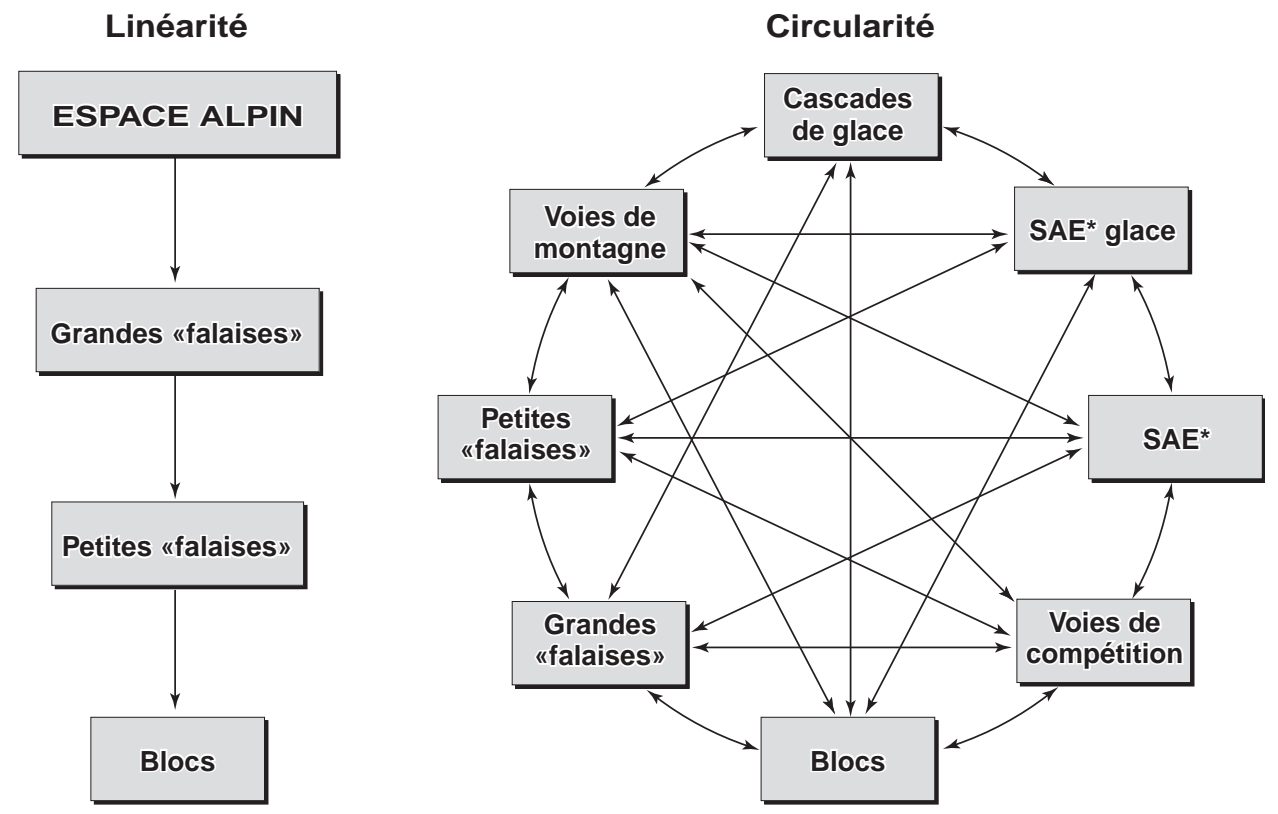


\section{Mythes, symboles, imaginaire}

Certains chercheurs étudient la symbolique des espaces et des éléments naturels selon les cultures sportives, invitant à sortir d'une approche strictement déterministe de l'espace pour expliquer la dynamique socio-spatiale des pratiques sportives. Ainsi, J.-P. Bozonnet (1992) montre la présence de déterminismes culturels qui organisent la relation entre la montagne et ses usagers et reposent avant tout sur le façonnage et le renouvellement de l'imaginaire autour de figures emblématiques de référence (imaginaire prométhéen, régénérateur, écologique). Les écrits de B. Jeu (1977) montrent par ailleurs que l'imaginaire construit sur l'espace participe à la constitution de figures sportives signifiantes sur le plan émotionnel.

\section{Une approche structuraliste des relations aux espaces sportifs de nature}

Dans une perspective similaire, les recherches de C. Pociello (1995) montrent que les relations à la nature ne peuvent se comprendre sans que soient pris en compte les déterminismes culturels qui président aux préférences pour certaines actions. Selon la réflexologie évoquée par G. Durand (1992), des processus anthropologiques prédisposeraient les garçons à l'appropriation de schèmes verticalisants et les filles à l'adoption de schèmes du blotissement. Ce phénomène expliquerait le marquage sexué des relations à l'espace où les hommes seraient sur-représentés dans les pratiques d'extérieur, notamment dans les espaces agressifs, tumultueux et incertains, alors que les femmes seraient largement consommatrices d'espaces intérieurs ou préféreraient un contact d'ordre plus contemplatif et bucolique avec les éléments naturels. De même, les différences stylistiques entre les alpinistes modernes (grimpe frontale) et les hédo-californiens post-modernes (grimpe sinueuse) s'expliqueraient par des prédispositions renvoyant à des schèmes éducatifs différents.

Mais il serait également possible de mettre en évidence le marquage social des usages de la nature à partir des différences culturelles entre les agents sociaux du public, qui privilégieraient dans les espaces de nature les pratiques écologiques et contemplatives, et les intervenants du privé qui préféreraient les pratiques motorisées et plus agressives (Pociello, 1981).

\section{UN NIVEAU D'INTERACTION FONCTIONNEL : LA SPATIALITÉ}

L'approche par la spatialité, bien que moins développée et moins explicitée que l'approche spatiale, constitue une deuxième entrée possible pour appréhender l'interaction entre cultures sportives et espaces de pratiques. Elle mène à observer comment se construisent les relations des pratiquants avec les sites naturels comme espaces d'action fonctionnels, en termes d'investissement géo-sportif, de fréquentation, de déplacements et de formes spécifiques d'usages. Une telle démarche réactive les théories de l'action dont le cadre théorique permet d'étudier la spatialité médiatrice, sans toutefois délaisser des entrées structurelles dans la mesure où la saisie des pratiques s'effectue de l'extérieur. 


\section{LES ENTRÉES STRUCTURELLES}

L'hypothèse d'une distribution des sites en fonction de la localisation géographique des centres urbains et touristiques d'où proviennent les pratiquants ou de la localisation des clubs et des différents acteurs qui s'investissent dans le plein air est séduisante. Mais J.-P. Augustin (1995) rappelle qu'une explication gravitaire standard est insuffisante pour comprendre les jeux de distance entre les individus et les sites de nature en regard de la diversité des moyens de déplacement et des variations proxémiques d'ordre culturel.

Il est cependant indéniable que les spatialités se transforment sous l'effet de variables structurelles et de différents types de médiations infrastructurelles, technologiques ou communicationnelles (Corneloup, 1993; Augustin, 2000). La construction d'infrastructures de transport (autoroutes, trains à grande vitesse, remontées mécaniques mises en réseau entre stations, etc.), le développement d'objets technologiques innovants (vélo tout terrain, parapente, parcours acrobatiques en hauteur, GPS, etc.), le jeu des médias (revues spécialisées et Internet), la mise en place de signaux et de balisages, la construction ou la rénovation des infrastructures d'hébergement, la création de nouveaux sites de pratique, notamment de sites situés dans l'entre-deux entre espaces aménagés et non aménagés (Bourdeau, 2003) participent à la recomposition des relations fonctionnelles qui structurent la spatialité des pratiquants.

\section{LES LOGIQUES SPATIALES DES SPORTIFS}

Il s'agit ici d'envisager les logiques pratiques à partir desquelles les personnes pratiquant des sports de montagne et de nature investissent les espaces récréatifs. En référence aux théories de l'action, les études sur les relations existantes entre les objets sportifs et les sportifs eux-mêmes s'efforcent de comprendre comment ces derniers investissent les pratiques et les objets sportifs de nature. Les travaux de J. Corneloup (1999) et de R. De Bellefon (2001) sur les processus d'ancrage dans le contexte de l'alpinisme montrent ainsi que la formation à ce sport passe le plus souvent par une initiation aux espaces de pratique et à la culture sportive de référence durant la jeunesse, faisant de l'immersion dans la nature et de diverses médiations fonctionnelles et symboliques deux processus moteurs pour l'activation des relations avec les espaces sportifs de la montagne. L'articulation étroite avec les processus qui relèvent d'une approche de la territorialité (voir infra) est ici manifeste, et les travaux analysés prennent implicitement en compte le continuum entre les registres d'interaction fonctionnel et existentiel.

\section{LE TERRITOIRE COMME CHAMP D'INTERACTION ORGANISATIONNEL ENTRE CULTURES SPORTIVES ET ESPACES DE PRATIQUES}

L'interaction entre les cultures sportives et les supports naturels peut aussi être abordée sous l'angle territorial, qui permet, d'une part, de comprendre de l'intérieur les logiques sociales et géographiques en jeu dans le façonnage des lieux et, $d$ 'autre part, d'étudier la singularité des espaces de pratique. En effet, les meso ou micro 
espaces ne sont pas équivalents, car ils acquièrent une identité en fonction du marquage qui prend forme au fil des interactions géo-sportives. Il s'impose alors de prendre en compte les contextes socio-géographiques pour comprendre la manière dont se vivent et se forment ces territoires, dans la mesure où les sportifs ne font pas que consommer l'espace, mais le marquent aussi par une projection d'appropriation et de sens.

\section{LA CONSTRUCTION SOCIALE DES TERRITOIRES DE PRATIQUE}

L'entrée dans le territoire ne se fait pas ici par l'espace, mais par le public ou les acteurs essayant de construire leurs territoires de pratique. On peut alors établir de nombreuses comparaisons pour montrer les décalages, les ressemblances et les différences entre différents groupes d'acteurs dans leur manière de construire leurs territoires de pratique. Les travaux de J. Corneloup (1999) ou ceux de C. Pociello (1995) ont, par exemple, montré que les espaces de pratique n'étaient pas consommés de la même façon par différents groupes, et que des modalités distinctes de pratique pour diverses activités (escalade, kayak, parapente, ski de fond...) permettent de déterminer des identités particulières associées à chaque site.

\section{Tensions territoriales et violence symbolique}

De manière globale, les infrastructures et la fréquentation des sites suscitent des tensions territoriales entre familles d'acteurs cherchant à réguler les entrées et à définir la bonne manière de pratiquer. Dans tous les sites emblématiques, comme à Chamonix, par exemple, les controverses ne manquent pas pour limiter ou augmenter le degré d'équipement des voies en fonction du style de jeu privilégié (Corneloup, 1999). Tout comme les oppositions entre usages récréatifs différents ou entre usages sportifs et professionnels - usages citadins et ruraux - de la nature sont fréquentes et trahissent la présence d'enjeux culturels autour de l'accès et de l'aménagement des espaces de pratique, des questions comme le balisage et l'ouverture de chemins ruraux, débattues entre cavaliers, randonneurs pédestres, vététistes et agriculteurs (Marsat, 2002), ou comme l'accès aux rivières, que les pêcheurs disputent aux adeptes du canyoning ou des sports d'eau-vive (Mounet, 1996), sont très représentatives de ces conflits d'intérêt.

Dans une autre perspective, les recherches de E. De Léséleulec (2000) invitent à comprendre comment un espace de pratique peut se transformer en territoire sportif sous l'action de grimpeurs locaux qui marquent de leur empreinte la culture d'un site. L'étude ethnosociologique réalisée par cet auteur sur un site d'escalade du sud de la France révèle le marquage territorial de cet espace qui se transforme en lieu, moyennant une forte régulation des formes d'échange au sein du groupe d'acteurs, et entre les acteurs et l'espace d'action. Au-delà de l'équipement particulier des voies d'escalade, qui exige un certain engagement psychologique par une distance prononcée entre les points d'assurage, c'est l'occupation culturelle du site par les locaux qui produit ce marquage territorial traduisant une violence symbolique exercée sur les grimpeurs « extérieurs » pour qu'ils respectent l'éthique et le style local de pratique. 
L'usage des sites de pratique s'inscrit donc dans un processus territorial complexe qui nécessite la prise en compte du façonnage stylistique des espaces de pratique. La neutralité géographique des sites n'est qu'apparente. Derrière la lecture spatiale des espaces de pratique se dévoile le marquage socio-géographique des sites, et la prise en compte des cultures sportives semble alors incontournable pour comprendre la manière dont les territoires émergent et se façonnent. Le recours aux notions de médiation territoriale (Augustin, 2000) et de haut-lieu (Micoud, 1991) semble alors tout à fait opportun pour comprendre les relations territorialisantes que les sportifs établissent avec les espaces de pratique.

\section{TERRITOIRES DE PRATIQUE ET DÉVELOPPEMENT}

Les processus de territorialisation peuvent aussi être analysés à partir des formes et des logiques de développement que les acteurs élaborent et dont ils imprègnent les objets géo-sportifs de nature. Si les travaux initiés par J.-P. Augustin (1994) montrent comment la pratique amateur du surf a participé à territorialiser la côte aquitaine, il demeure que le jeu des professionnels est tout aussi déterminant. À cet égard, les recherches de P. Bourdeau (1991) contribuent à la compréhension du marquage territorial des espaces alpins par les acteurs emblématiques que sont les guides de haute montagne. Le déploiement de l'identité sociale et culturelle de ce groupe professionnel passe en effet par une mise en forme organisationnelle et symbolique de ses espaces d'activité. Ces espaces deviennent alors des territoires construits sur un enchevêtrement de spatialités complexes et multiscalaires qu'unifient de multiples processus de marquage symbolique et identitaire effectués au prix de nombreux conflits internes et externes qui portent sur la définition légitime de la pratique professionnelle et de ses relations - culturelles, réglementaires, économiques, politiques - avec d'autres groupes professionnels comme les moniteurs de ski, les accompagnateurs en montagne ou les moniteurs d'escalade. L'évolution des cultures professionnelles des métiers sportifs de la montagne ne manque évidemment pas d'interagir avec cette dynamique tensionnelle (Corneloup et Bourdeau, 2001).

\section{Formes et logiques de développement}

Les recherches entreprises par un groupe de travail issu des sciences et techniques des activités physiques et sportives (STAPS) (Corneloup et al., 2001) montrent quant à elles l'existence de formes territoriales qui organisent le développement des sites de pratique et de logiques territoriales identifiables par la manière dont les professionnels et l'ensemble des acteurs impliqués investissent les espaces sportifs de nature. La forme qu'épousera un site de pratique apparaît alors comme le produit d'un équilibre entre différentes forces exogènes et endogènes qui contribuent à lui donner une orientation managériale. Et en fonction de différents processus mis en action - économique, écologique et politique - et reposant sur des principes d'action identifiables - vision de la nature, approche du lieu, éthique sportive, rationalité économique, conventions partagées et processus décisionnels adaptés -, il est possible d'observer des formes de développement qui marquent de leur empreinte la gestion des sites. Les territoires qui en émergent renvoient donc à des conceptions et à des identités territoriales différentes en fonction du jeu des acteurs et des publics engagés dans cette économie locale des rôles. 
Mais au-delà du marquage local autour d'une forme emblématique, il faut reconnaître que les territoires sportifs sont l'objet de luttes et de conflits entre acteurs aux intérêts divergents. Les écrits d'O. Hoibian (2000) montrent ainsi comment la montagne a suscité pendant plus d'un siècle nombre de conflits entre les différents acteurs de l'alpinisme (Club alpin français - CAF, Fédération française de la montagne et de l'escalade - FFM, Groupe de haute montagne - GHM...) et les différents modèles de diffusion des sports de montagne : modèle « bourgeois » et nationaliste pour le CAF, modèle élitiste (au sens social et sportif) pour le GHM et modèle sportif pour la FFM, s'opposant sur les pratiques sportives et sur la manière légitime de parcourir les voies d'ascensions.

\section{LES TERRITORIALITÉS DE PRATIQUE : VERS UNE INTERACTION D'ORDRE EXISTENTIEL}

Dans cette dernière perspective, on cherche à saisir les processus par lesquels les individus construisent leurs relations avec un territoire. L'étude des territorialités permet ainsi de comprendre comment les individus ou des micro-groupes s'approprient et s'identifient à un territoire.

\section{L'APPROCHE STRUCTURELLE DES TERRITORIALITÉS SPORTIVES}

Il est possible de classer dans la catégorie des approches structurales de la territorialité - «par le haut, abstraite » (Di Méo, 1998) - les études portant sur les facteurs macro-géographiques et sociologiques qui participent à la construction de la relation entre l'adepte d'un sport et son espace de pratique. La démarche consiste alors à déterminer et à saisir les processus qui concourent à la définition des usages des lieux par le pratiquant. À partir d'approches quantitatives ou spéculatives, la construction d'un modèle de lecture de la territorialité permet, par exemple, d'expliquer la transformation des usages de l'espace d'escalade par l'apparition de nouveaux points d'assurance, tout comme on peut montrer que la production récente de topoguides thématiques ou interactifs, à la manière des guides de l'Institut géographique National (IGN), a pour effet de modifier les pratiques touristico-sportives en redonnant, notamment, de l'importance à des territorialités en réseau (Corneloup, 1999).

\section{LES TERRITORIALITÉS EN ACTES : L'APPROCHE INTERACTIONNELLE}

Mais la manière d'analyser le processus d'ancrage à un (ou des) territoire(s) de pratique peut aussi reposer sur l'examen des facteurs micro-sociologiques et géographiques qui permettent de saisir la territorialité interactionnelle - «par la base, vécue, émotionnelle » (Di Méo, 1998) - du pratiquant sur son site de pratique. Dans ce cas, l'approche est davantage ethnographique et vise à comprendre les relations entre le territoire vécu, perçu, rêvé et la territorialité en situation. Telle est la démarche esquissée par Michel Favory (J.-P. Augustin et al., 1994) à propos des néo-territorialités des surfeurs aquitains. C'est aussi en analysant les interactions entre le milieu et les usages corporels que E. Boutroy (2002) fait ressortir les propriétés spécifiques de la pratique des via ferrata par rapport à l'escalade sportive. 
Une approche phénoménologique des pratiques peut également permettre de saisir l'expérience vécue et les interactions de proximité qui contribuent à l'élaboration des pratiques sportives et de leurs espaces. De multiples variables interactionnelles (la fréquentation sociale des sites, les conditions climatiques, la forme physique du moment, le contexte psycho-social, la sensibilité aux lieux...) ponctuent sans cesse le quotidien de l'action; sans oublier le poids que peut représenter l'expérience personnelle du site, les relations affectives établies ou évitées, les évènements vécus, les relations réelles et imaginaires avec les voies ou les parcours que l'on a réussis ou échoués, qui participent de l'individualisation des territorialités.

En abordant la territorialité des ouvreurs de voies d'alpinisme dans le massif des Écrins comme un entre-deux structurel et interactionnel, les travaux de P. Bourdeau (2002) soulignent la complémentarité de ces deux registres d'approche de la territorialité : alors que l'approche cartographique associée à une base de données permet de saisir la dimension structurelle de la territorialité des ouvreurs en fonction de différents processus d'ancrage dans un espace d'action très sélectif, l'approche par les récits d'ascension, les "stratégies » et les savoirfaire d'ouverture, basée quant à elle sur des entretiens ou des récits autobiographiques, permet d'aborder la dimension interactionnelle de cette territorialité.

\section{CONCLUSION}

Envisagée ici à partir d'un état de la recherche française ${ }^{5}$, la question de $l^{\prime}$ interaction entre cultures sportives et espaces de pratique des sports de nature mobilise un large éventail d'entrées disciplinaires (histoire, sociologie, ethnologie, géographie, écologie, droit), heuristiques (espace, spatialité, territoire, territorialité) et méthodologiques (approches structurelles, interactionnelles). Cette diversité correspond à une complémentarité et à une gradation fécondes des échelles géographiques et sociologiques prises en compte (du local au global, du micro au macro, de l'individu au groupe), ainsi qu'en ce qui concerne les registres d'interaction concernés (physique, fonctionnel, organisationnel, existentiel).

Au final, que cette interaction soit envisagée sous l'angle du façonnage des cultures sportives par un espace-support qui oriente - sinon détermine - les propriétés formelles des pratiques, ou bien au contraire sous l'angle de la production par les cultures sportives de formes géo-sportives fonctionnelles (sites, pistes, itinéraires, voies...), c'est bien la notion de co-construction temporalisée des cultures sportives et de leurs espaces d'action qui se dégage de l'ensemble du corpus étudié. À cet égard, si la dimension spatiale de ce processus occupe une place centrale dans la grille de lecture proposée, sa dimension temporelle n'en reste pas moins incontournable. En effet, non seulement l'« épaisseur » temporelle joue un rôle fondamental dans l'inter-construction des cultures et des territoires sportifs, mais de plus la dynamique des permanences et des changements qui les anime est particulièrement active. 
En effet, face aux multiples facteurs de changement relevés par la recherche - hybridation des cultures sportives montagnardes et non montagnardes, irruption de références urbaines, technologisation des activités et des supports de pratique, mobilité élargie et accrue au sein des espaces de pratique, recherche croissante d'hédonisme, etc. -, la question des permanences dans l'interaction entre les cultures sportives et les espaces de pratique ne saurait être éludée. Les travaux récents de J. Corneloup (2002) sur les cultures sportives de montagne le soulignent en mettant en évidence le classicisme des valeurs et des images associées aux pratiques, parmi lesquelles font figure de lignes de force l'attachement aux fondements moraux et éthiques de l'alpinisme et à la notion d'effort, le respect et l'humilité vis-à-vis de la nature ou encore l'adhésion très limitée aux nouvelles modalités médiatisées et aux nouveaux espaces marchandisés des pratiques que sont respectivement le surf des neiges tout terrain (free-ride) et les parcs de loisirs sportifs.

Dans le même registre, les travaux de synthèse de J.-P.Augustin (2002) montrent de manière convaincante que les phénomènes de mouvement et de changement observés au sein du système géo et socio-sportif participent davantage d'une recomposition que d'une rupture radicale. De fait, après une phase fondatrice de rupture ostentatoire vis-à-vis de la culture sportive traditionnelle, les modalités de pratique, d'organisation et de spatialisation des sports de montagne et de nature font presque systématiquement l'objet de processus d'intégration et de «normalisation » (médiatisation, compétition, commercialisation, institutionnalisation, standardisation technique) qui animent une dialectique du changement et de la permanence. Là encore, quels qu'en soient les échelles et les registres de saisie, l'interaction entre cultures sportives et espaces de pratique constitue un champ d'observation particulièrement fécond pour étudier les médiations socio-territoriales qu'assurent les pratiques récréatives entre l'ici urbain et l'ailleurs montagnard ou naturel (Piolle et al., 1992).

\section{NOTES}

1 La notion de culture sportive retenue ici - proche de l'acception anglophone de subculture - intègre aussi bien les pratiques, techniques, savoir-faire et usages du corps, de la nature, du matériel... que les relations, les références fondatrices, l'histoire, les valeurs, l'éthique et les codes (vestimentaires, langagiers...) qui caractérisent les différentes activités ou disciplines inscrites dans l'« univers » des sports de montagne.

2 Cette typologie reprend les trois catégories proposées par M. Le Berre (Le Berre, 1992), et leur ajoute le registre «fonctionnel » correspondant à la spatialité. Son application aux sports de nature a déjà été proposée par Jean-Pierre Augustin (J.-P. Augustin et al., 1994).

3 Le terme de « falaise » d'escalade, bien qu'incorrect dans une acception géographique stricte, est conservé ici dans la mesure où il est utilisé dans la culture sportive concernée.

4 Itinéraire d'ascension équipé de barreaux, agrès et câbles pour faciliter et sécuriser la progression et l'accès à la verticalité d'un public qui n'est pas toujours autonome en escalade rocheuse. Nées dans les Dolomites (Italie) pendant la Première Guerre mondiale, les via ferrata se sont diffusées tardivement mais très rapidement dans les Alpes françaises à partir du début des années 1990.

5 Cette perspective est évidemment très restreinte et gagnerait à être étendue à une échelle géographique et linguistique plus ambitieuse. Un des objectifs de cet article est donc de susciter des réactions permettant de développer des échanges internationaux autour de la question traitée. 


\section{BIBLIOGRAPHIE}

AUGUSTIN, Jean-Pierre et al. (1994) Surf Atlantique. Les territoires de l'éphémère. Bordeaux, MSHA.

(1995) Sport, géographie et aménagement. Paris, Nathan.

(2000) Activités ludo-sportives et médiations territoriales. Dans Montagnes Méditerranéennes, 11: 29-32.

(2002) La diversification territoriale des activités sportives. Dans L'année sociologique, 52 (2) : 417-435.

AUGUSTIN, Jean-Pierre et al. (1994) Surf Atlantique. Les territoires de l'éphémère. Bordeaux, MSHA.

BOURDEAU, Philippe (1991) Guides de haute montagne : territoire et identité. Grenoble, Revue de géographie alpine (Coll. "Ascendances").

- (1998) Les Alpes comme terrain de jeu de l'Europe. Dans Rapport sur l'État des Alpes, Commission Internationale Pour la Protection des Alpes (CIPRA), Edisud, Aix-en Provence, pp. 252-259.

(2002) Temporalités et territorialités de pratique de l'alpinisme. Deux siècles de premières ascensions dans le massif des Écrins (XIXe et XXe siècles). Dans Olivier Hoibian et Jacques Defrance (dir.) Deux siècles d'alpinismes européens, Paris, L'Harmattan, pp. 189-217.

- (2003) Territoires du hors-quotidien : une géographie culturelle du rapport à l'ailleurs dans les sociétés urbaines contemporaines; le cas du tourisme sportif de montagne et de nature. Rapport de diplôme d'habilitation à diriger des recherches, Université Joseph Fourier, Grenoble.

BOURDEAU, Philippe et MAO, Pascal (2002) « Nouvelles » pratiques sportives de nature, nouveaux espaces... nouveaux territoires? Une problématique géoculturelle de l'accès aux espaces naturels. Dans L'accès du public aux espaces naturels et forestiers; outils d'analyse et méthodes de gestion, Paris, Hermès-Lavoisier, pp. 345-364.

BOUTROY, Éric (2002) Une technique du vertige? Les usages du corps dans une pratique ascensionniste : la via ferrata. Dans Techniques et Culture, 39 : 25-32.

BOZONNET, Jean-Paul (1992) Des monts et des mythes, l'imaginaire social de la montagne. Grenoble, Presses Universitaires de Grenoble.

CHAZAUD, Pierre (2000) Les logiques du tourisme sportif face aux stratégies de développement du territoire Dans Montagnes Méditerranéennes, 11 : 49-54.

CHIVALLON, Christine (1998) Projet de groupe de recherche sur l'espace anthropologique, réponse à l'appel d'offre " aide à projet nouveau » du CNRS, Maison des sciences de l'Homme d'Aquitaine, Bordeaux.

CORNELOUP, Jean (1993) Escalades et société; contribution à l'analyse du système, du communicationnel et du social, thèse de doctorat, Université Paris XI.

- (1999) Management de la nature, des lieux et des pratiques sportives. Dans Pour une valorisation des milieux naturels, Paris, Belin.

- (2002) Les théories sociologiques de la pratique sportive. Paris, Presses Universitaires de France.

CORNELOUP, Jean et BOURDEAU, Philippe (2001) Culture professionnelle et métiers du tourisme sportif de montagne, Téoros, 20 (3) : 32-43.

DEBARBIEUX, Bernard (1995) Tourisme et montagne. Paris, Économica. 
DE BELLEFON, Renaud (2001) Paroles de guides... Identités de montagnards et d'alpinistes, Babel, $5:$ 81-102.

DE LÉSÉLEUC, Éric (2000) «Voler » et Donner... Ethnosociologie d'un lieu anthropologique: le site d'escalade de Claret, Université Montpellier 1.

DIÉNOT, Josy et THEILLER, Didier (1999) Les nouveaux loisirs sportifs en montagne. Bordeaux, MSHA.

DI MÉO, Guy (1998) Géographie sociale et territoires. Paris, Nathan.

DUPUY, Nicolas et MAO, Pascal (2000) Vers un diagnostic des territoires de pratiques sportives de pleine nature. Dans Montagnes Méditerranéenne, 11 : 17-26.

DURAND, Gilbert (1992) Les structures anthropologiques de l'imaginaire. Paris, Dunod.

FRANCHINI S. et LEMPERIÈRE, G. (2000) Recherche d'indicateurs biologiques pertinents dans l'évaluation des perturbations liées à la pratique de la descente de canyons en Ardèche. Dans Montagnes méditerranéennes, no 11, Mirabel.

GRIFFOND-BOITIER, Anne (1995) Le niveau d'équipement sportif, reflet d'une hiérarchie urbaine. Dans Le sport et ses espaces, Éditions du CTHS, Paris, pp. 13-25.

HAUMO(NT, Antoine (1995) Les variations géographiques du sport. Dans Sport, relations sociales et action collective, Bordeaux, MSHA, pp. 51-59

HOIBIAN, Olivier (2000) Les alpinistes en France. Paris, L'Harmattan.

JEU, Bernard (1977) Le sport, l'émotion, l'espace. Paris, Vigot.

LE BERRE, Maryvonne (1992) Territoires. Dans Bailly et al., Encyclopédie de géographie, Paris, Economica, pp. 617-638.

LORET, Alain (1995) Génération glisse, dans l'eau, l'air, la neige..., la révolution du sport des « années fun». Paris, Autrement (série « Mutation»).

LORET, Alain et al. (1993) Sport et management. Paris, Dunod.

MAO, Pascal (2003) Les lieux de pratique sportives de nature dans les espaces ruraux et montagnards. Contribution à l'analyse de l'espace géographique du sport. Grenoble, Institut de Géographie Alpine, thèse de doctorat de l'Université Joseph Fourier.

MARSAT, Jean-Bernard (2002) Les acteurs du tourisme dans la gestion de l'accès aux espaces naturels : l'exemple de deux activités de pleine nature dans un même territoire. Dans L'accès du public aux espaces naturels et forestiers; outils d'analyse et méthodes de gestion, Paris, Hermès-Lavoisier, pp. 309-325.

MATHIEU, Daniel et PRAICHEUX, Jean (1987) Sports en France. Paris, Fayard-Reclus.

MICOUD, André (1991) Des Hauts-lieux. Paris, Éditions du CNRS.

MIZRAHI, Robert et BOURGES, Pierre (1979) La vision de la nature chez les pratiquants de loisirs de montagne. Paris, CORDES.

MOUNET, Jean-Pierre (1996) Sports d'eau vive et pêche en rivière : un conflit asymétrique, STAPS, $40: 4-20$.

MOUNET, Jean-Pierre, NICOLLET, Jean-Pierre et ROCHEBLAVE, Mathieu, (2000) L'impact des activités sportives de nature sur l'environnement naturel, Montagnes Méditerranéennes, $11: 67-76$.

PIGEASSOU, Jean-Charles (2001) Dynamiques, mutations et changements dans le sport. Dans Montagnes Méditerranéennes, 13: 19-26.

PIOLLE, Xavier et al. (1992) Pratique de la montagne et société urbaine. Dans Dossiers de la revue de Géographie Alpine, 7.

POCIELLO, Christian (1995) Les cultures sportives. Paris, Presses Universitaires de France. SERVOIN, François (1993) La commune de montagne. Paris, Economica. 